\title{
Coherence of an electron spin in quantum dots generated by a resonant optical pulse with elliptic polarization
}

\author{
Shinichi Tomimoto*, Keisuke Kawana, Akira Murakami, Yasuaki Masumoto \\ Institute of Physics, University of Tsukuba, Tsukuba 305-8571, Japan
}

\begin{abstract}
We have experimentally observed the spin polarization process of single electrons in InP/InGaP quantum dots by time-resolved Kerr rotation measurements. It is found that the inversion of the spin polarization direction occurs with the variation of the intensity of the optical pulse. The spin coherence lifetime abruptly changes on the occurrence of the inversion. We have reproduced the inversion in numerical simulations using the density operator of the electron-trion four-level system, assuming a small deviation of the optical pulse from circular polarization. The change of the spin lifetime is attributed to the qualitative change of the four-level system in the electric polarization state.
\end{abstract}

Keywords: electron spin, polarization, InP quantum dot, Kerr rotation

\section{Introduction}

The two spin states of an electron with $s=1 / 2$ in a semiconductor quantum dot (QD) make up the space of the operational states of a quantum bit (qubit) [1]. This solid-state qubit has an excellence of the integratability with existing semiconductor technologies. The lifetime of the spin states in QDs is generally very long (up to microseconds) [2] owing to the suppression of the relaxation mechanisms coming from the spin-orbit coupling [3]. They can be initialized and manipulated by optical field, which is resonant or near-resonant to the electron-trion (negatively-charged exciton) transitions [4]. The initialization is equivalent to the generation of coherence between the two spin states, and its fastest process is obtained by the resonant excitation by circularly-polarized light. According to the optical selection rule, the $\sigma+$ excitation transfers some of the probability amplitude of the electron state $|z\rangle$ to that of the trion state $|T z\rangle$ (here $z$ is the growth axis of self-assembled QDs which is antiparallel to the optic axis), which results in the net electron spin polarization along $-z[5,6,7]$.

Here we have experimentally observed the spin polarization process of single electrons in InP/InGaP QDs by time-resolved Kerr rotation (TRKR) measurements. It is found that the inversion of the spin polarization direction occurs by the change of the pump intensity, and that the spin lifetime abruptly changes on the occurrence of the inversion. We discuss these findings by means of numerical simulations using the density operator of the electron-trion four-level system. It suggests that a small deviation of the optical pulse from circular polarization is essential for the inversion. The change of the spin lifetime is at-

\footnotetext{
${ }^{*}$ Corresponding author

Email address: tomimoto.shinichi.ft@u.tsukuba.ac.jp (Shinichi Tomimoto)
}

tributed to the qualitative change of the four-level system in the electric polarization state.

The outline of this article is as follows. In Sec. 2, we note the calculation model which is used in the discussion. The experimental observations are explained in Sec. 3 and discussed in Sec. 4 by means of the model calculation. The summary of this article is given in Sec. 5 .

\section{Calculation model}

\subsection{Basic Hamiltonian}

In what follows, we confine ourselves to consideration of the QD electron-trion system in a magnetic field $B$ along the $x$ axis, which is affected by the classical field of light traveling along $-z$ (Voigt geometry). This situation is depicted in Fig. 1(a). We assume single electrons with spin $s=1 / 2$ in self-assembled QDs with the growth direction $z$ consisting of zincblende materials. We denote the two energy eigenstates of the electron in the magnetic field $B \| x$ by $|x\rangle=|0\rangle$ and $|\bar{x}\rangle=|1\rangle$. Then the photoexcited trion is made of a hole, having a total angular momentum of $3 / 2$, and a singlet pair of electrons. We consider only the lowest states of the trion. As they are made principally of the two heavy-hole states $\left|m_{z}= \pm 3 / 2\right\rangle$, we can define a pseudospin space to describe them $\left(s_{T}=1 / 2\right)$, which has two energy eigenstates $|T x\rangle=|2\rangle$ and $|T \bar{x}\rangle=|3\rangle$ in the field $B$. Thus we can construct the four-level system represented in Fig. 1(b). The angular frequencies $\omega_{e}$ and $\omega_{h}$ are defined so as to represent the Zeeman splittings of the electron and trion, respectively: $2 \hbar \omega_{e}=g_{e} \mu_{\mathrm{B}} B$ and $2 \hbar \omega_{h}=g_{h} \mu_{\mathrm{B}} B$. Here $\mu_{\mathrm{B}}$ is the Bohr magneton, $g_{e}$ and $g_{h}$ are $g$ factors (in the field of $B \| x$ ) of the electron and trion (heavy hole), respectively. We assume they have positive values in the level configuration of Fig. 1(b). 

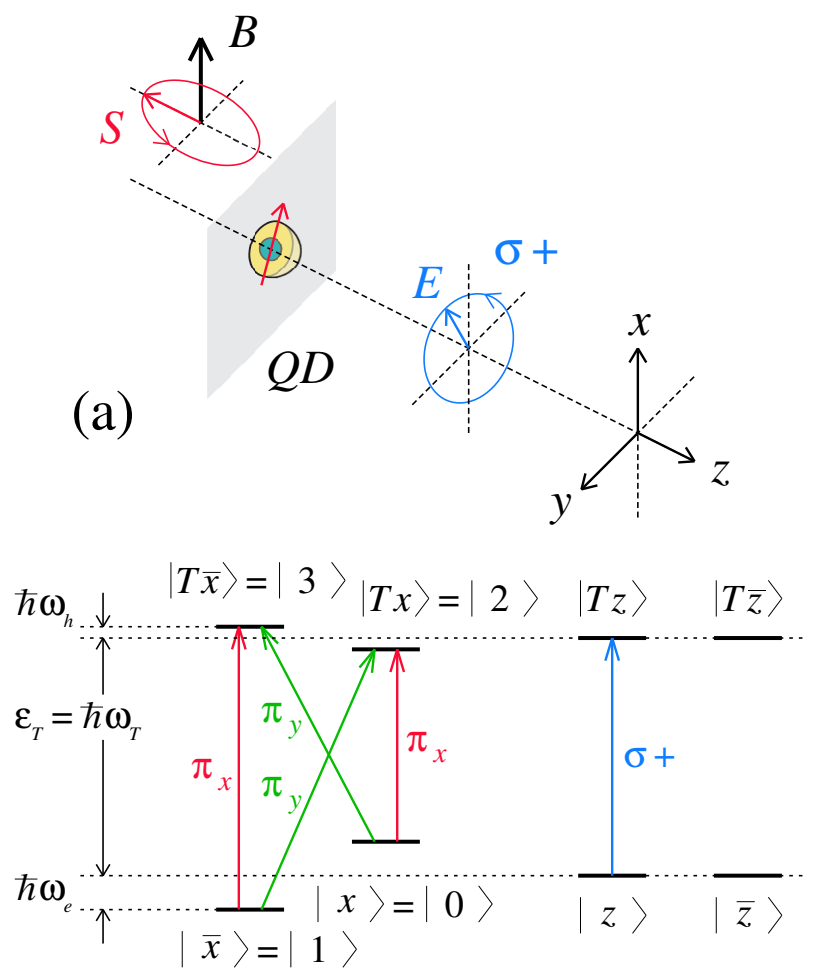

(b)

(c)

Figure 1: (Color online) (a) Schematic representation of the system under consideration. The $z$ axis is defined to be along the growth direction of the QD, in which one conduction electron is present. The optical pulse traveling along $-z$ induces the polarization of the electron spin $S$, which subsequently precesses around the magnetic field $B \| x$ perpendicular to the $z$ axis (Voigt geometry). The rotating electric field of a $\sigma+$-polarized pulse is expected to induce the spin polarization along $-z$, according to the optical selection rule. (b) Energy eigenstates of the four-level system consisting of the spin states of the electron $|x\rangle=|0\rangle,|\bar{x}\rangle=|1\rangle$ and those of the trion (negatively-charged exciton) $|T x\rangle=|2\rangle,|T \bar{x}\rangle=|3\rangle$ in the field of $B \| x$. (c) The same spin states shown in the basis of the $z$ axis.

The unperturbed part of the Hamiltonian of the four-level system is expressed as

$$
\begin{aligned}
\mathcal{H}_{0}= & \hbar \omega_{e}|x\rangle\left\langle x\left|-\hbar \omega_{e}\right| \bar{x}\right\rangle\left\langle\bar{x}\left|+\left(\varepsilon_{T}-\hbar \omega_{h}\right)\right| T x\right\rangle\langle T x| \\
& +\left(\varepsilon_{T}+\hbar \omega_{h}\right)|T \bar{x}\rangle\langle T \bar{x}|,
\end{aligned}
$$

where $\varepsilon_{T}=\hbar \omega_{T}$ is the energy separation between the electron and trion levels at $B=0$.

In the dipole approximation, the vertical and cross transitions in the four-level system of Fig. 1(b) can be represented by the dipole moments $\mu$ and $i \mu$ which are coupled to optical electric fields of orthogonal linear polarizations $\pi_{x}$ and $\pi_{y}$, respectively. As these oscillating moments are $\pi / 2$ out of phase with each other (at $B=0$ ), we have the latter include the phase factor $i$. Then, in the rotating wave approximation, the interaction part of the Hamiltonian is given by

$$
\begin{aligned}
\mathcal{V}= & -\hbar \Omega_{x}(t)\left\{e^{-i \omega_{0} t}|T x\rangle\left\langle x\left|+e^{i \omega_{0} t}\right| x\right\rangle\langle T x|\right\} \\
& -i \hbar \Omega_{y}(t)\left\{-e^{-i\left(\omega_{0} t+\delta\right)}|T x\rangle\left\langle\bar{x}\left|+e^{i\left(\omega_{0} t+\delta\right)}\right| \bar{x}\right\rangle\langle T x|\right\} \\
& -\hbar \Omega_{x}(t)\left\{e^{-i \omega_{0} t}|T \bar{x}\rangle\left\langle\bar{x}\left|+e^{i \omega_{0} t}\right| \bar{x}\right\rangle\langle T \bar{x}|\right\}
\end{aligned}
$$

$$
-i \hbar \Omega_{y}(t)\left\{-e^{-i\left(\omega_{0} t+\delta\right)}|T \bar{x}\rangle\left\langle x\left|+e^{i\left(\omega_{0} t+\delta\right)}\right| x\right\rangle\langle T \bar{x}|\right\} .
$$

Here $\omega_{0}$ is the central frequency of the optical pulse, $\delta$ is the relative phase between the $\pi_{x}$ - and $\pi_{y}$-polarized waves, $\Omega_{x}(t)$ and $\Omega_{y}(t)$ are time-dependent real optical Rabi frequencies for the respective polarizations. The basic Hamiltonian of the system under consideration is the sum of Eqs. (1) and (2),

$$
\mathcal{H}=\mathcal{H}_{0}+\mathcal{V}
$$

We assume a hyperbolic-secant pulse envelope [4, 8]

$$
\Omega_{0}(t)=\Omega \operatorname{sech}(\kappa t),
$$

where $\kappa$ is the bandwidth of the pulse, and $\Omega$ is a real Rabi amplitude. Then we assume the time-dependent Rabi frequencies to be

$$
\begin{aligned}
& \Omega_{x}(t)=\Omega_{0}(t) \cos \theta, \\
& \Omega_{y}(t)=\Omega_{0}(t) \sin \theta,
\end{aligned}
$$

where $\tan \theta$ determines the ratio $\Omega_{y} / \Omega_{x}$ [9], which is the ratio of the amplitudes of $\pi_{x}$ - and $\pi_{y}$-polarized optical electric fields. By tuning the values of $\delta$ and $\theta$, we can obtain an arbitrary polarization state of the incident pulse. In the case of $\theta=\pi / 4$ $\left(\Omega_{x}=\Omega_{y}\right), \delta=-\pi / 2$ gives the circular polarization of $\sigma+$, which connects the states $|z\rangle=(|x\rangle+|\bar{x}\rangle) / \sqrt{2}$ and $|T z\rangle=(|T x\rangle+$ $|T \bar{x}\rangle) / \sqrt{2}$ while the states $|\bar{z}\rangle=(|x\rangle-|\bar{x}\rangle) / \sqrt{2}$ and $|T \bar{z}\rangle=(|T x\rangle-$ $|T \bar{x}\rangle) / \sqrt{2}$ are decoupled from this pulse field.

The Hamiltonian of Eq. (3) is the variation of the existing models $[8,9,10]$, which deal with coherent optical rotations of the electron spin $[4,11,12]$.

\subsection{Equation of motion}

Using the Hamiltonian of Eq. (3), we compute the time evolution of the density operator $\rho(t)$ of the four-level system by solving the quantum Liouville equation

$$
i \hbar \frac{d}{d t} \rho(t)=[\mathcal{H}, \rho(t)]
$$

For numerical calculation, it is preferable to obtain the equation in the interaction picture removing the fast-oscillating terms with $\omega_{0}$ or $\omega_{T}$. With this view, we define the operator $\sigma(t)$ by

$$
\rho(t)=\exp \left[-\frac{i}{\hbar} \mathcal{H}_{0} t\right] \sigma(t) \exp \left[\frac{i}{\hbar} \mathcal{H}_{0} t\right]
$$

Replacing $\rho(t)$ in Eq. (6) with this expression, we obtain the equation for $\sigma(t)$

$$
\frac{d}{d t} \sigma(t)=\left[\mathcal{V}_{\mathrm{R}}, \sigma(t)\right]
$$

where the operator $\mathcal{V}_{\mathrm{R}}$ is defined by

$$
i \hbar \mathcal{V}_{\mathrm{R}}=\exp \left[\frac{i}{\hbar} \mathcal{H}_{0} t\right] \mathcal{V} \exp \left[-\frac{i}{\hbar} \mathcal{H}_{0} t\right]
$$


The specific form of $\mathcal{V}_{\mathrm{R}}$ is

$$
\begin{aligned}
\mathcal{V}_{\mathrm{R}}= & i \Omega_{x}(t)\left\{e^{-i \omega_{1} t}|T x\rangle\left\langle x\left|+e^{i \omega_{1} t}\right| x\right\rangle\langle T x|\right\} \\
& -\Omega_{y}(t)\left\{-e^{-i\left(\omega_{3} t+\delta\right)}|T x\rangle\left\langle\bar{x}\left|+e^{i\left(\omega_{3} t+\delta\right)}\right| \bar{x}\right\rangle\langle T x|\right\} \\
& +i \Omega_{x}(t)\left\{e^{-i \omega_{4} t}|T \bar{x}\rangle\left\langle\bar{x}\left|+e^{i \omega_{4} t}\right| \bar{x}\right\rangle\langle T \bar{x}|\right\} \\
- & \Omega_{y}(t)\left\{-e^{-i\left(\omega_{2} t+\delta\right)}|T \bar{x}\rangle\left\langle x\left|+e^{i\left(\omega_{2} t+\delta\right)}\right| x\right\rangle\langle T \bar{x}|\right\} .
\end{aligned}
$$

Here the frequencies are

$$
\begin{aligned}
& \omega_{1}=\omega_{0}+\omega_{e}-\omega_{T}+\omega_{h}, \\
& \omega_{2}=\omega_{0}+\omega_{e}-\omega_{T}-\omega_{h}, \\
& \omega_{3}=\omega_{0}-\omega_{e}-\omega_{T}+\omega_{h}, \\
& \omega_{4}=\omega_{0}-\omega_{e}-\omega_{T}-\omega_{h} .
\end{aligned}
$$

As far as we consider resonant or near-resonant cases $\left(\omega_{0} \simeq\right.$ $\omega_{T}$ ), fast-oscillating terms are absent in $\mathcal{V}_{\mathrm{R}}$.

\subsection{Dissipation processes}

In order to consider the time evolution of the electron spin coherence for a long time period, we need to include dissipative processes in the model. We can define longitudinal and transverse relaxations between any two states of the four-level system of Fig. 1(b). Among them, we consider the following processes which appears to be important for the electron spin coherence : (a) the longitudinal relaxation from either of the trion states $(|2\rangle,|3\rangle)$ to that of the electron states $(|0\rangle,|1\rangle)$ which is mainly due to spontaneous emission (the rate $1 / T_{1}=2 \Gamma$ ), (b) the transverse relaxations (decoherence) between the trion states $(2 \Gamma)$ and between the respective pair of electron and trion states $(\Gamma)$, which arise from the decrease of the trion population due to (a) the spontaneous emission, (c) the pure decoherences between the trion states $\left(\gamma_{T 2}\right)$ and between the electron states $\left(\gamma_{e 2}\right)$ which we call spin depolarization here, and (d) spontaneously generated coherence (SGC) which may accompany the spontaneous emission $\left(\Gamma_{c}=\Gamma\right)[5,14]$.

Meanwhile, we do not cover (e) the longitudinal relaxations between the trion states $\left(1 / T_{T 1}\right)$ and between the electron states $\left(1 / T_{e 1}\right)$, i.e. spin-flip, as they seem to be less important than (or their effect may be included in) the spin depolarization. We also neglect (f) any decoherence processes between the electron and trion other than (b), for simplicity.

\subsubsection{Spontaneous emission}

The relaxations (a) and (b) coming from the trion decay are summarized as the terms

$$
\begin{aligned}
\mathcal{L}[\rho]=-i \hbar \sum_{j, k} \Gamma_{j, k}\{|j\rangle\langle j| \rho & +\rho|j\rangle\langle j| \\
& -|k\rangle\langle j|\rho| j\rangle\langle k|\} .
\end{aligned}
$$

which is placed in the right side of Eq. (6). The state $|j\rangle$ is a trion state $(|2\rangle$ or $|3\rangle)$, and $|k\rangle$ is an electron state $(|0\rangle$ or $|1\rangle)$. We assume all the rates $\Gamma_{j, k}$ to be the same $(=\Gamma)$.

The $\mathcal{L}[\rho]$ includes the trion decay of the rate $2 \Gamma$

$$
\mathcal{L}[\rho]_{j j}=\langle j|\mathcal{L}[\rho]| j\rangle=-2 i \hbar \Gamma \rho_{j j},
$$

and the population recovery of the electrons

$$
\mathcal{L}[\rho]_{k k}=+i \hbar \Gamma\left(\rho_{22}+\rho_{33}\right) .
$$

It also includes the decoherences accompanying the trion decay

$$
\mathcal{L}[\rho]_{j k}=-i \hbar \Gamma \rho_{j k}
$$

and

$$
\mathcal{L}[\rho]_{23}=-2 i \hbar \Gamma \rho_{23} .
$$

The Eq. (15) is for the respective pair of electron and trion states, and Eq. (16) is for the trion spin coherence. In contrast, $\mathcal{L}[\rho]$ does not affect the electron spin coherence,

$$
\mathcal{L}[\rho]_{01}=0,
$$

since it allows only for the incoherent transitions.

For the interaction picture, we define the operator $\mathcal{L}[\sigma]$ by

$$
\begin{aligned}
i \hbar \mathcal{L}[\sigma]= & \exp \left[\frac{i}{\hbar} \mathcal{H}_{0} t\right] \mathcal{L}[\rho] \exp \left[-\frac{i}{\hbar} \mathcal{H}_{0} t\right] \\
= & -i \hbar \sum_{j, k} \Gamma_{j, k}\{|j\rangle\langle j|\sigma+\sigma| j\rangle\langle j| \\
& -|k\rangle\langle j|\sigma| j\rangle\langle k|\} .
\end{aligned}
$$

Then $\mathcal{L}[\sigma]$ can be added to the right side of Eq. (8).

\subsubsection{Spin depolarization}

The relaxation (c) is expressed by

$$
\begin{aligned}
\mathcal{D}[\rho]= & -i \hbar\left\{\gamma_{e 2}(|1\rangle\langle 1|\rho| 0\rangle\langle 0|+| 0\rangle\langle 0|\rho| 1\rangle\langle 1|)\right. \\
& \left.+\gamma_{T 2}(|3\rangle\langle 3|\rho| 2\rangle\langle 2|+| 2\rangle\langle 2|\rho| 3\rangle\langle 3|)\right\}
\end{aligned}
$$

to be placed in the right side of Eq. (6), which includes the pure decoherence of the trion spin of the rate $\gamma_{T 2}$

$$
\mathcal{D}[\rho]_{23}=-i \hbar \gamma_{T 2} \rho_{23},
$$

and that of the electron spin of the rate $\gamma_{e 2}$

$$
\mathcal{D}[\rho]_{01}=-i \hbar \gamma_{e 2} \rho_{01} .
$$

For the interaction picture, we define the operator $\mathcal{D}[\sigma]$ by

$$
\begin{aligned}
i \hbar \mathcal{D}[\sigma]= & \exp \left[\frac{i}{\hbar} \mathcal{H}_{0} t\right] \mathcal{D}[\rho] \exp \left[-\frac{i}{\hbar} \mathcal{H}_{0} t\right] \\
= & -i \hbar\left\{\gamma_{e 2}(|1\rangle\langle 1|\sigma| 0\rangle\langle 0|+| 0\rangle\langle 0|\sigma| 1\rangle\langle 1|)\right. \\
& \left.+\gamma_{T 2}(|3\rangle\langle 3|\sigma| 2\rangle\langle 2|+| 2\rangle\langle 2|\sigma| 3\rangle\langle 3|)\right\}
\end{aligned}
$$

Then $\mathcal{D}[\sigma]$ can be added to the right side of Eq. (8).

\subsection{3. $S G C$}

In a $\Lambda$-type three-level system, the spontaneous decay of the highest state may create a coherent superposition of the two lower ones in case their energy splitting is smaller than the radiative linewidth. This process is called SGC (spontaneously generated coherence) $[13,14]$. Considering SGC in the present case of the four-level system [Fig. 1(b)], the trion decay of the process (a) may affect the electron spin coherence when the Zeeman splitting of the lower states $|0\rangle$ and $|1\rangle$ is small. 
To take the effect into account, we append the term

$$
i \hbar \Gamma_{c}\{\langle T z|\rho| T z\rangle-\langle T \bar{z}|\rho| T \bar{z}\rangle\}=i \hbar \Gamma_{c}\left(\rho_{32}+\rho_{23}\right)
$$

to the right side of Eq. (6) for the components $\rho_{01}$ and $\rho_{10}$, following the manner of Economou et al. [14]. For this purpose, we define a new operator

$$
C[\rho]=i \hbar \Gamma_{c}\left(\rho_{32}+\rho_{23}\right)\{|0\rangle\langle 1|+| 1\rangle\langle 0|\} .
$$

Then the operator $C[\sigma]$ for the right side of Eq. (8) in the interaction picture is given by

$$
i \hbar C[\sigma]=\exp \left[\frac{i}{\hbar} \mathcal{H}_{0} t\right] C[\rho] \exp \left[-\frac{i}{\hbar} \mathcal{H}_{0} t\right]
$$

It has the following explicit form

$$
\begin{aligned}
C[\sigma]=2 \Gamma_{c} & \operatorname{Re}\left(e^{-2 i \omega_{h} t} \sigma_{32}\right) \\
& \times\left\{e^{2 i \omega_{e} t}|0\rangle\left\langle 1\left|+e^{-2 i \omega_{e} t}\right| 1\right\rangle\langle 0|\right\} .
\end{aligned}
$$

\subsubsection{Master equation}

Considering the above dissipation terms, we obtain the master equation for the density operator $\rho(t)$,

$$
i \hbar \frac{d}{d t} \rho(t)=[\mathcal{H}, \rho(t)]+\mathcal{L}[\rho]+\mathcal{D}[\rho]+C[\rho],
$$

from Eq. (6) for the present four-level system. This is equivalent to the equation for $\sigma(t)$,

$$
\frac{d}{d t} \sigma(t)=\left[\mathcal{V}_{\mathrm{R}}, \sigma(t)\right]+\mathcal{L}[\sigma]+\mathcal{D}[\sigma]+C[\sigma]
$$

which is obtained from Eq. (8) in the interaction picture. As $\sigma$ is a Hermitian operator $\left(\sigma=\sigma^{\dagger}\right)$, there are ten independent matrix elements $\sigma_{i j}$ on the basis set of four vectors, $|0\rangle,|1\rangle,|2\rangle$, and $|3\rangle$. Consequently, Eq. (28) constructs a set of ten coupled first-order differential equations on $\sigma_{i j}(t)$. is

In the present model, the spin decoherence rate for the trion

$$
1 / T_{T 2}=2 \Gamma+\gamma_{T 2},
$$

as obtained from Eqs. (16) and (20). Meanwhile, it is

$$
1 / T_{e 2}=\gamma_{e 2}
$$

for the electron [Eqs. (17) and (21)] if we neglect the effect of SGC. The presence of SGC makes the situation a little more complicated.

\subsection{Mean values}

To trace the time evolution of the four-level system, we use two kinds of parameters as reference indices. One is the mean values of spin components, and the other is those of electric polarization components.

\subsubsection{Spin components}

Here we summarize the relations of the mean values of spin components to the density matrix elements. For the electron spin $s$, they are

$$
\begin{aligned}
\left\langle s_{x}\right\rangle & =\operatorname{Tr}\left(\rho s_{x}\right) \\
& =\left(\rho_{00}-\rho_{11}\right) / 2=\left(\sigma_{00}-\sigma_{11}\right) / 2, \\
\left\langle s_{y}\right\rangle & =\operatorname{Tr}\left(\rho s_{y}\right) \\
& =\{\langle y|\rho| y\rangle-\langle\bar{y}|\rho| \bar{y}\rangle\} / 2 \\
& =i\left(\rho_{10}-\rho_{01}\right) / 2=-\operatorname{Im}\left(e^{2 i \omega_{e} t} \sigma_{10}\right),
\end{aligned}
$$

and

$$
\begin{aligned}
\left\langle s_{z}\right\rangle & =\operatorname{Tr}\left(\rho s_{z}\right) \\
& =\{\langle z|\rho| z\rangle-\langle\bar{z}|\rho| \bar{z}\rangle\} / 2 \\
& =\left(\rho_{10}+\rho_{01}\right) / 2=\operatorname{Re}\left(e^{2 i \omega_{e} t} \sigma_{10}\right) .
\end{aligned}
$$

In the same way, for the trion pseudospin $\boldsymbol{s}_{T}$, they are

$$
\begin{aligned}
\left\langle s_{T x}\right\rangle & =\operatorname{Tr}\left(\rho s_{T x}\right) \\
& =\left(\rho_{22}-\rho_{33}\right) / 2=\left(\sigma_{22}-\sigma_{33}\right) / 2, \\
\left\langle s_{T y}\right\rangle & =\operatorname{Tr}\left(\rho s_{T y}\right) \\
& =\{\langle T y|\rho| T y\rangle-\langle T \bar{y}|\rho| T \bar{y}\rangle\} / 2 \\
& =-\operatorname{Im} \rho_{32}=-\operatorname{Im}\left(e^{-2 i \omega_{h} t} \sigma_{32}\right),
\end{aligned}
$$

and

$$
\begin{aligned}
\left\langle s_{T z}\right\rangle & =\operatorname{Tr}\left(\rho s_{T z}\right) \\
& =\{\langle T z|\rho| T z\rangle-\langle T \bar{z}|\rho| T \bar{z}\rangle\} / 2 \\
& =\operatorname{Re} \rho_{32}=\operatorname{Re}\left(e^{-2 i \omega_{h} t} \sigma_{32}\right) .
\end{aligned}
$$

\subsubsection{Induced electric polarization}

To represent the dipole moments $\mu$ and $i \mu$ mentioned in Sec. 2.1, we define the Hermitian operators $p_{x}$ and $p_{y}$,

$$
\begin{aligned}
& p_{x}=\mu\{|3\rangle\langle 1|+| 1\rangle\langle 3|+| 2\rangle\langle 0|+| 0\rangle\langle 2|\}, \\
& p_{y}=i \mu\{|3\rangle\langle 0|-| 0\rangle\langle 3|+| 2\rangle\langle 1|-| 1\rangle\langle 2|\} .
\end{aligned}
$$

Then the electric polarization induced optically in a QD is given by the density matrix elements as

$$
\begin{aligned}
\left\langle p_{x}\right\rangle & =\operatorname{Tr}\left(\rho p_{x}\right) \\
& =\mu\left(\rho_{20}+\rho_{31}+\rho_{02}+\rho_{13}\right) \\
=2 \mu \operatorname{Re} & {\left[e^{-i \omega_{T} t}\left\{e^{i\left(\omega_{h}+\omega_{e}\right) t} \sigma_{20}+e^{-i\left(\omega_{h}+\omega_{e}\right) t} \sigma_{31}\right\}\right] }
\end{aligned}
$$

for the $\pi_{x}$, and

$$
\begin{aligned}
\left\langle p_{y}\right\rangle & =\operatorname{Tr}\left(\rho p_{y}\right) \\
& =i \mu\left(-\rho_{30}-\rho_{21}+\rho_{12}+\rho_{03}\right) \\
=2 \mu \operatorname{Im} & {\left[e^{-i \omega_{T} t}\left\{e^{i\left(-\omega_{h}+\omega_{e}\right) t} \sigma_{30}+e^{i\left(\omega_{h}-\omega_{e}\right) t} \sigma_{21}\right\}\right] }
\end{aligned}
$$

for the $\pi_{y}$ polarization direction. Then we define the polarization amplitudes $P_{x}(t)$ and $P_{y}(t)$ by

$$
\begin{gathered}
i P_{x}(t)=e^{i\left(\omega_{h}+\omega_{e}\right) t} \sigma_{20}+e^{-i\left(\omega_{h}+\omega_{e}\right) t} \sigma_{31}, \\
i P_{y}(t)=e^{i\left(-\omega_{h}+\omega_{e}\right) t} \sigma_{30}+e^{i\left(\omega_{h}-\omega_{e}\right) t} \sigma_{21},
\end{gathered}
$$


so that

$$
\begin{gathered}
\left\langle p_{x}\right\rangle=2 \mu \operatorname{Re}\left[e^{-i \omega_{T} t} \cdot i P_{x}(t)\right], \\
\left\langle p_{y}\right\rangle=2 \mu \operatorname{Re}\left[-i e^{-i \omega_{T} t} \cdot i P_{y}(t)\right] .
\end{gathered}
$$

From Eq. (41), we can see that $\left\langle p_{x}\right\rangle$ and $\left\langle p_{y}\right\rangle$ consist of the fast oscillation part at the frequency $\omega_{T}$ and the slowly-varying amplitude $P_{x}(t)$ or $P_{y}(t)$. As a consequence, we can express the polarization amplitude by a complex vector,

$$
\boldsymbol{P}(t)=P_{x}(t) \boldsymbol{e}_{x}-i P_{y}(t) \boldsymbol{e}_{y},
$$

including the phase factor, in the unit of $2 \mu$. Here $\boldsymbol{e}_{x}$ and $\boldsymbol{e}_{y}$ are the unit vectors along the $\pi_{x}$ and $\pi_{y}$ directions, respectively.

This polarization amplitude can also be expressed by two circular components. For this purpose, we define a new set of bases,

$$
\begin{aligned}
& \boldsymbol{e}_{+}=\left(\boldsymbol{e}_{x}+i \boldsymbol{e}_{y}\right) / \sqrt{2}, \\
& \boldsymbol{e}_{-}=\left(\boldsymbol{e}_{x}-i \boldsymbol{e}_{y}\right) / \sqrt{2} .
\end{aligned}
$$

Then, from Eq. (42), we obtain

$$
\boldsymbol{P}(t)=P_{+}(t) \boldsymbol{e}_{+}+P_{-}(t) \boldsymbol{e}_{-},
$$

where the two circular amplitudes are given as

$$
\begin{aligned}
& P_{+}(t)=\left\{P_{x}(t)-P_{y}(t)\right\} / \sqrt{2}, \\
& P_{-}(t)=\left\{P_{x}(t)+P_{y}(t)\right\} / \sqrt{2} .
\end{aligned}
$$

These components should become the source of the circularlypolarized coherent radiation at the frequency $\omega_{T}$ emitted from the QDs after the experience of the incident optical pulse.

\section{Experimental results}

We have experimentally observed the optical spin polarization process by picosecond time-resolved Kerr rotation (TRKR) measurements of single electrons in InP QDs. The experimental details are described elsewhere [15]. The sample is chargetunable InP QDs embedded in $\operatorname{~}_{0.5} \mathrm{Ga}_{0.5} \mathrm{P}$ barriers grown on an $\mathrm{n}^{+}$-GaAs substrate $[16,17,18,19]$. By applying an appropriate bias voltage $(U=-0.175 \mathrm{~V})$ along the crystal growth axis $z$, an ensemble of QDs with one residual electron each on average is prepared [20]. This sample is mounted in a magneto-optical cryostat (sample temperature $T=5 \mathrm{~K}$ ), and the TRKR is observed by the degenerate pump-and-probe method in transverse magnetic field $B=1 \mathrm{~T}$. The pump (probe) photon energy is $1.746 \mathrm{eV}$, which is resonant to the electron-trion transition in the QDs. The time resolution of the measurement is about $2 \mathrm{ps,}$ which is determined by the pulse width of the light source, a mode-locked Ti:sapphire laser (repetition rate $82 \mathrm{MHz}$ ).

Figure 2 shows the time evolution of the TRKR signal and its dependence on the pump intensity $I$. The oscillation with a period of 47 ps comes from the spin precession of single electrons in QDs. Its frequency corresponds to the known electron $g$ factor of $g_{e}=1.52$ in the QDs $[16,17,18]$, from which we can identify the origin. Although this oscillation can be seen at any of the three pump intensities $I$ in Fig. 2, it shows rather

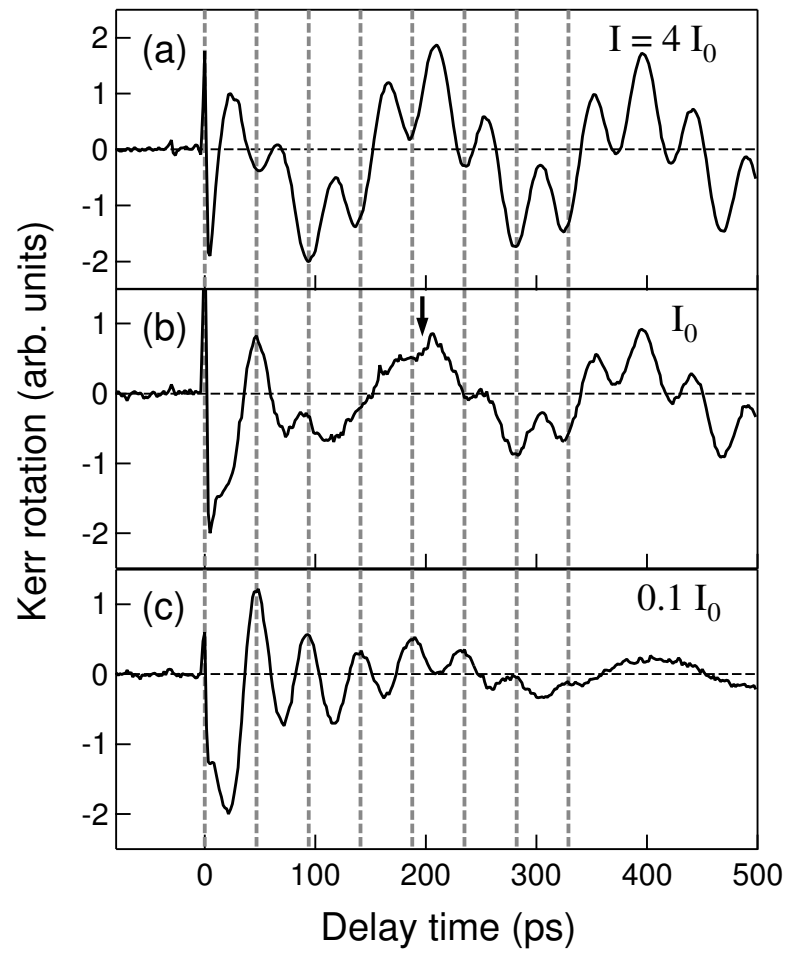

Figure 2: Experimental data of TRKR in the InP QDs, and its dependence on the pump intensity $I\left(I_{0}=250 \mathrm{~nJ} / \mathrm{cm}^{2}\right)$ at $B=1 \mathrm{~T}$ and $T=5 \mathrm{~K}$. The vertical broken lines show the temporal points of the oscillation peaks in (c).

complicated dependence on $I$. The most interesting point is the phase reversal which can be noticed by comparing the data at (a) $I=4 I_{0}$ and (c) $0.1 I_{0}\left(I_{0}=250 \mathrm{~nJ} / \mathrm{cm}^{2}\right)$. We can see the oscillation bottoms in (a) at the temporal points of the vertical broken lines correspond to the oscillation peaks in (c). This phase reversal means that the spin polarization direction is inverted only by changing the pump intensity. Furthermore, we find that the lifetime of the oscillation in (c) (about $200 \mathrm{ps)} \mathrm{is}$ much shorter than that in (a) (longer than $1 \mathrm{~ns}$ ), though the frequency remains unchanged suggesting that the origin of the oscillation is not altered (single electrons in QDs). At the intermediate pump intensity (b) $I=I_{0}$, we can see the competition of the two features observed in (a) and (c), which results in the temporal disappearance of the oscillation around $200 \mathrm{ps}$ as denoted by an arrow. In (a) and (b), another low-frequency oscillation with a period of $177 \mathrm{ps}$ is obvious. This originates from the electron spins in the GaAs substrate, and is not important here.

\section{Discussion}

In this section, we try to explain the experimental observations which shows the above intriguing behavior of the spin polarization of single electrons by means of numerical simulations. We obtain the solutions $\sigma(t)$ by solving Eq. (28) numerically by the standard Runge-Kutta method. In the calculation, we assume the physical parameters to reproduce the experimental situation of the TRKR measurement of the InP QDs 
mentioned in the previous section. The $g$ factors of the electron and trion (heavy hole) in the field of $B \| x$ are assumed to be $g_{e}=1.52$ and $g_{h}=0.068$, respectively $[16,17,18]$. The electron-trion transition energy at $B=0$ is $\varepsilon_{T}=\hbar \omega_{T}=1.746$ $\mathrm{eV}$. The trion recombination lifetime $T_{1}=1 / 2 \Gamma$ is $250 \mathrm{ps}$ [19]. The relaxation rate $\Gamma_{c}$ is equal to $\Gamma$ or zero when the SGC effect is taken into consideration or not, respectively [14]. The times for the pure spin decoherences are $1 / \gamma_{e 2}=1 / \gamma_{T 2}=2 \mathrm{~ns}$. The properties of the optical pulse is determined by five parameters $\left(\omega_{0}, \Omega, \kappa, \delta, \theta\right)$, but we deal only with the resonant cases $\omega_{0}=\omega_{T}$ below. As the thermal energy at $T=5 \mathrm{~K}$ exceeds the electron Zeeman splitting at $B=2 \mathrm{~T}$, we assume the initial state of unpolarized electron spins: at $t=-\infty, \rho_{00}=\rho_{11}=1 / 2$ $\left(\sigma_{00}=\sigma_{11}=1 / 2\right)$, all other elements of $\rho(\sigma)$ are zero.

First we discuss the origin of the phase reversal with the variation of $I$ seen in Fig. 2. This means the change of sign in $\left\langle s_{z}\right\rangle$, as we observe the time dependence of $\left\langle s_{z}\right\rangle$ in the TRKR measurements. The pump pulse in the experiment is circularlypolarized nominally. Therefore we assume the circular polarization of $\sigma+(\theta=\pi / 4, \delta=-\pi / 2)$ here for a while. Figure 3(a1) shows the calculated trajectory of the mean values of the electron spin components $\overrightarrow{S_{e}}(t)=\left(\left\langle s_{y}\right\rangle,\left\langle s_{z}\right\rangle\right)$ from $t=-50$ to 150 ps at $B=1 \mathrm{~T}$. Here we assume the optical bandwidth of $\kappa=\kappa_{0}=0.88 \times 10^{12} \mathrm{~s}^{-1}$ corresponding to the experimental pulse temporal width of 2 ps. The figure shows the data for a pulse peak intensity, i.e. the Rabi frequency $\Omega=1.5$ $\left[10^{12} \mathrm{rad} \cdot \mathrm{s}^{-1}\right]$. The trajectory starts from $\left\langle s_{y}\right\rangle=\left\langle s_{z}\right\rangle=0$. Then, during the duration of the optical pulse, $\left\langle s_{z}\right\rangle$ oscillates approximately between 0 and $-1 / 4$ showing the Rabi oscillation, whose number of cycles increases with $\Omega$. The negative sign of $\left\langle s_{z}\right\rangle$ is natural, considering the optical selection rule as mentioned before. The spin precession around the magnetic field $(B \| x)$ begins subsequently, which depicts the circular trajectory shown in the figure. This results in the oscillation of $\left\langle s_{z}\right\rangle$ which is observed in the TRKR measurements. When the optical polarization is $\sigma+$, it always starts from a negative value as seen in Fig. 3(a2) regardless of $\Omega$.

Interestingly enough, $\left\langle s_{y}\right\rangle$ does not remain zero during the Rabi oscillation, and $\overrightarrow{S_{e}}(t)$ traces the leaf-shaped trajectory seen in Fig. 3(a1). First, $\overrightarrow{S_{e}}(t)$ grows to the direction $\left\langle s_{y}\right\rangle>0$ (and $\left\langle s_{z}\right\rangle\langle 0)$. After returning to the origin, it grows to $\left\langle s_{y}\right\rangle<0$ next. Then, via the origin, it grows again to the direction $\left\langle s_{y}\right\rangle>0$, and so on. Thus the trajectory draws the two leaf-shaped closed loops in $\left\langle s_{y}\right\rangle>0$ and $\left\langle s_{y}\right\rangle<0$ alternately. The appearance of the two loops is characteristic of the case of narrow bandwidth $\kappa$ in comparison with the electron Zeeman splitting. Figure 3(b1) shows the trajectory at $B=0$, which coincides with the $\left\langle s_{z}\right\rangle$ axis thoroughly, and the two loops are absent. The angle between them opens symmetrically to the $\left\langle s_{z}\right\rangle$ axis when $\kappa$ decreases [Figs. 3(c1), (c2)], or $B$ increases [(d1), (d2)]. The starting point of the spin precession is somewhere in these two loops, and it depends on the pulse area sensitively. Although the spin precession begins from the right loop in Fig. 3(a1), it can begin from the left one at different $\Omega$. This suggests the oscillation phase of $\left\langle s_{z}\right\rangle$ in the spin precession slightly depends on $\Omega$. But its amount is too small in our experimental condi-
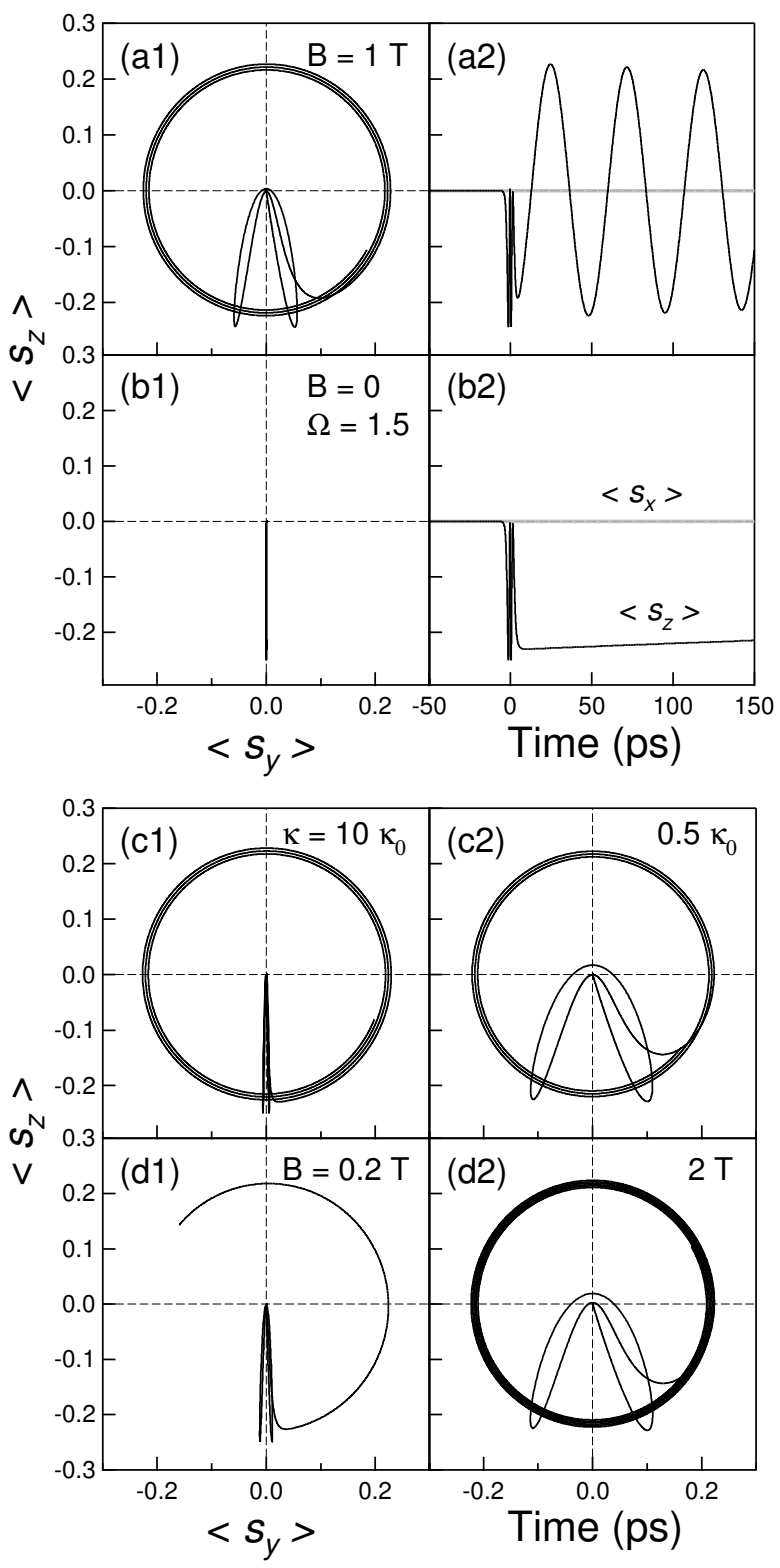

Figure 3: Calculated spin mean values at (a) $B=1 \mathrm{~T}$ and (b) $B=0 \mathrm{~T}(\Omega=1.5$ $\left.\left[10^{12} \mathrm{rad} \cdot \mathrm{s}^{-1}\right]\right)$. The left panels show the trajectories of $\overrightarrow{S_{e}}(t)$ from $t=-50$ to $150 \mathrm{ps}$. The time dependences of $\left\langle s_{z}\right\rangle$ (black) and $\left\langle s_{x}\right\rangle$ (gray) are shown in the right panels. The dependence of the trajectory on $\kappa(B=1 \mathrm{~T})$ and $B\left(\kappa=\kappa_{0}\right)$ is shown in (c) and (d), respectively. In (c1) and (c2), $\Omega$ is varied so as to keep the pulse area constant. (The value of $\Omega / \kappa$ is fixed.) Here we assume the optical polarization of $\sigma+$ and $\Gamma_{c}=0$. 
tion to explain the phase reversal seen in Fig. 2. Moreover, as our TRKR experiments are on ensemble QDs which have an inhomogeneity in transition dipoles, we have not been able to observe the Rabi oscillation directly.

As far as the ideal circular polarization is assumed, it is difficult to explain the phase reversal. Therefore we show next the data when the finite deviation from $\sigma+$ is assumed. Figure 4 shows the data when the polarization ellipticity is assumed to be $\theta=1.02 \times \pi / 4$. This makes $\Omega_{y}$ slightly larger than $\Omega_{x}$ (Eq. 5). This deviation from $\sigma+$ enables the optical coupling between $|\bar{z}\rangle$ and the trion states, which give rise to the phase reversal as below. The data at $\Omega=1$ and 15 are shown in (a) and (b), respectively. Although the effect of the deviation from $\sigma+$ is not significant at $\Omega=1$, it causes essentially different behavior at $\Omega=15$. With increase of the Rabi cycles, the turn-around point of $\left\langle s_{y}\right\rangle \sim 0$ denoted by arrows in (a1) and (b1) shifts in the positive direction of $\left\langle s_{z}\right\rangle$. As a result, the spin precession comes to start from positive $\left\langle s_{z}\right\rangle$, giving rise to the phase reversal [(a2), (b2)]. This does not occur in the case of $\theta=\pi / 4$. In practical experiments, the small deviation of $\Omega_{x}$ and $\Omega_{y}$ can come from a slight difference in the dipole moments for the $\pi_{x}$ and $\pi_{y}$ polarizations.

Next, we discuss the cause of the difference in the spin lifetime seen in Fig. 2 between (a) $I=4 I_{0}$ and (c) $0.1 I_{0}$. The spin lifetime is usually determined uniquely by the pure spin decoherence rate $\gamma_{e 2}$ [Eq. (30)]. The experimental data, however, suggests that the lifetime changes abruptly on the occurrence of the phase reversal. The longer lifetime at $I=4 I_{0}$ (more than $1 \mathrm{~ns})$ seems to be determined by $\gamma_{e 2}$. The abrupt change of the lifetime cannot be explained even when we include the effect of SGC. In Figs. 4(a2) and (b2), the time dependences of $\left\langle s_{z}\right\rangle$ in the case of $\Gamma_{c}=\Gamma$ (gray lines) with the effect of SGC are shown, and they are compared with those of $\Gamma_{c}=0$ (thin black lines) without SGC. We can see that SGC makes no significant effect on $\left\langle s_{z}\right\rangle$ in both the cases of $\Omega=1$ and 15 .

This is due to the precession of the electron spin in the magnetic field, which transfers the population alternately between the states $|z\rangle$ and $|\bar{z}\rangle$ as represented in Fig. 4(e) where the sizes of circles express the level populations. We assume here that the populations in the trion states are frozen because of the very small $g$ factor $g_{h}$. Then the decay of the trion from $|T z\rangle$ to $|z\rangle$ decreases the electron spin polarization when the population majority is in the $|\bar{z}\rangle$ state [the case $\alpha$ in Fig. 4(e)], but increases it when the majority is in the $|z\rangle$ state (the case $\beta$ ). Thus the net effect of SGC dissipates because it is the process accompanying the spontaneous emission which can occur whether the majority resides in $|z\rangle$ or $|\bar{z}\rangle$. The radiative process which has a considerable effect on the electron spin coherence needs to occur selectively in the situation of $\alpha$ or $\beta$. One of the candidates of such a process is coherent light scattering. Then we look into the electric polarization as a source of coherent radiation.

Figures 4(c) and (d) show the time dependence of the electric polarization components at $\Omega=1$ and 15 , respectively. As seen in $(\mathrm{c} 1)$ and $(\mathrm{d} 1)$, both the components $P_{x}(t)$ and $P_{y}(t)$ are real quantities, as their imaginary parts (gray lines) are negligibly small. This is a general feature of the resonant case $\left(\omega_{0}=\omega_{T}\right)$. Two circular components $P_{+}(t)$ and $P_{-}(t)$ are also real accord-
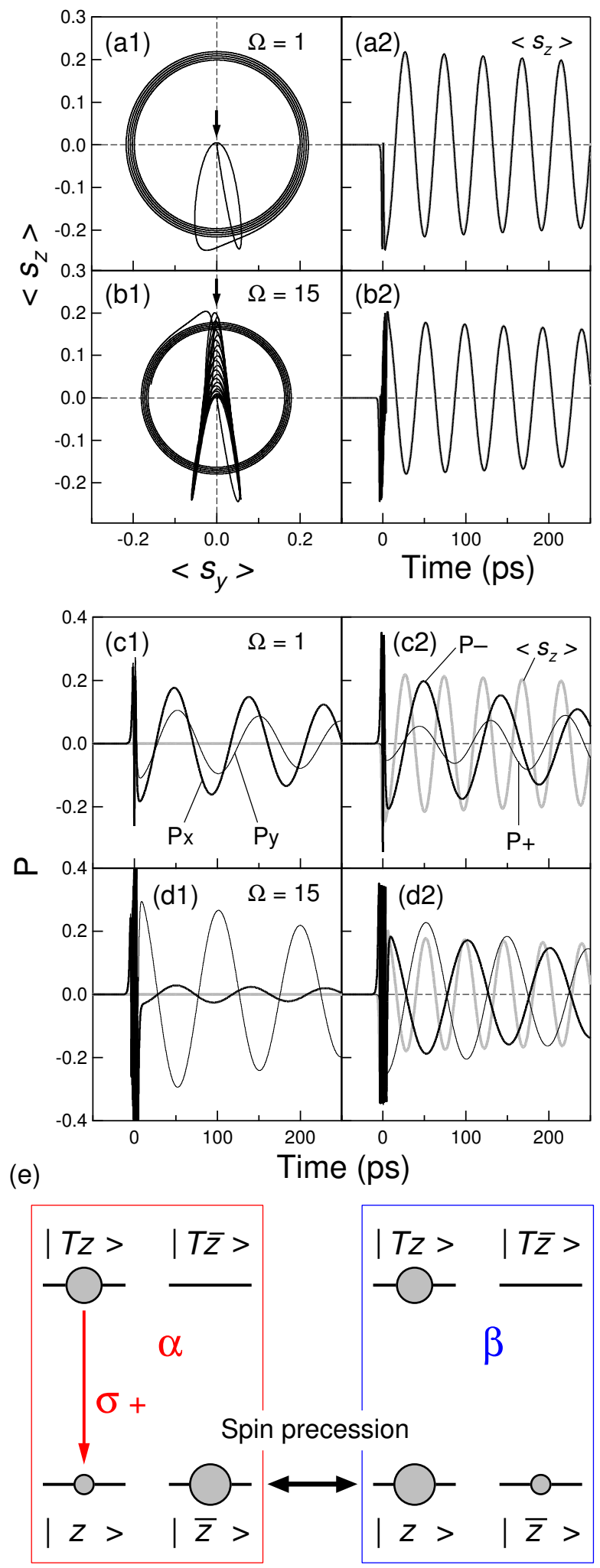

Figure 4: (Color online) (a)(b) Dynamics of spin components at $\Omega=1$ and 15 when a small deviation from $\sigma+$ is assumed $(\theta=1.02 \times \pi / 4, B=1 \mathrm{~T})$. In (a2) and (b2), the data of $\Gamma_{c}=\Gamma$ are also shown as gray lines which overlaps approximately with those of $\Gamma_{c}=0$. (c)(d) Electric polarization components corresponding to (a) and (b). (e) Schematic diagram of the four-level system. 
ingly. They are shown in (c2) and (d2) with $\left\langle s_{z}\right\rangle$. They exhibit slow oscillations with twice the period of the electron spin precession. Regardless of $\Omega, P_{-}(t)$ starts from its maximum amplitude at $t \sim 0$ and show cosine-like damped oscillation. The dynamics of $P_{+}(t)$ is rather different between $\Omega=1$ and 15, however. At $\Omega=1, P_{+}(t)$ starts from a small amplitude which gradually increases. Thus $P_{-}$dominates $P_{+}$at $t \leq 100 \mathrm{ps}$. This is the result of the fact that $P_{-}$is the co-circular component with the rotating electric field of $\sigma+$ of the excitation pulse. Thus the dominant $P_{-}$component can become the source of the coherent radiation emission of $\sigma+$. At $\Omega=15$, however, the $P_{-}$and $P_{+}$have comparable amplitudes even at $t \leq 100 \mathrm{ps}$. This results in the quasi-linear electric polarization along $y$ as seen in (d1). This is a consequence of the assumed deviation from $\sigma+$ $(\theta=1.02 \times \pi / 4)$. The difference of $P$ between $\Omega=1$ and 15 can cause the different spin lifetimes.

At $\Omega=1$, the $\sigma+$ coherent radiation emission swells when the absolute value of $P_{-}$comes close to extrema. Interestingly enough, those temporal points coincides approximately with the minima of $\left\langle s_{z}\right\rangle(t)$ [Fig. 4(c2)]. At these points, therefore the situation $\alpha$ in Fig. 4(e) is established, where the population majority resides in $|\bar{z}\rangle$, and simultaneously the $\sigma+$ coherent radiation is expected. This $\sigma+$ emission will transfer the population from $|T z\rangle$ to $|z\rangle$, and act destructively on the electron spin polarization. Meanwhile in the situation $\boldsymbol{\beta}$, the $\sigma+$ emission is switched off because the maximum points of $\left\langle s_{z}\right\rangle(t)$, where the majority is in $|z\rangle$, correspond approximately to vanishing points of $P_{-}(t)$ in Fig. 4(c2). In this way, although $\boldsymbol{\alpha}$ and $\boldsymbol{\beta}$ appear alternately due to the spin precession, the $\sigma+$ emission comes of $P_{-}$in $\alpha$ selectively. This may lead to the fast decay of the electron spin polarization observed at the low pump intensity in Fig. 2(c). At the high pump intensity [ $I=4 I_{0}$, Fig. 2(a)], this process is not effective because there is not a dominant circular component of $P$ in Fig. 4(d2). In this way, the change of the spin lifetime between $I=4 I_{0}$ and $0.1 I_{0}$ in Fig. 2 is tentatively attributed to the qualitative change of the $P$ state in the four-level system.

Although the pump pulse in the experiment is circularlypolarized nominally, we assume that the slight deviation of $\theta$ from the ideal value of $\pi / 4$ may arise from finite difference of either the transition dipole moments or optical electric-field amplitudes for the $\pi_{x}$ and $\pi_{y}$ polarizations. They are inevitable to some degree in the actual experimental situation and QDs. It will, however, be possible for us to control the optical ellipticity purposely, which may result in the variation of the critical pump intensity for the spin inversion. It seems interesting but is left for the future study at present.

\section{Summary}

We have experimentally observed the spin polarization process of single electrons in InP QDs by TRKR measurements. It is found that the inversion of the spin polarization direction occurs by the change of the pump intensity, and that the spin lifetime abruptly changes on the occurrence of the inversion. The numerical simulation using the density operator of the electrontrion four-level system has reproduced the inversion assuming a small deviation from circular polarization of the optical pulse. The change of the spin lifetime is attributed to the qualitative change of the four-level system in the electric polarization state.

\section{References}

[1] Semiconductor Spintronics and Quantum Computation, edited by D. D. Awschalom, D. Loss, and N. Samarth (Springer, Berlin, 2002).

[2] For example, A. Greilich, D. R. Yakovlev, A. Shabaev, Al. L. Efros, I. A. Yugova, R. Oulton, V. Stavarache, D. Reuter, A. Wieck, and M. Bayer, Science 313, 341 (2006).

[3] A. V. Khaetskii, and Y. V. Nazarov, Phys. Rev. B 61, 12639 (2000).

[4] Sophia E. Economou, and T. L. Reinecke, Phys. Rev. Lett. 99, 217401 (2007).

[5] M. V. Gurudev Dutt, Jun Cheng, Bo Li, Xiaodong Xu, Xiaoqin Li, P. R. Berman, D. G. Steel, A. S. Bracker, D. Gammon, Sophia E. Economou, Ren-Bao Liu, and L. J. Sham, Phys. Rev. Lett. 94, 227403 (2005).

[6] A. Greilich, R. Oulton, E. A. Zhukov, I. A. Yugova, D. R. Yakovlev, M. Bayer, A. Shabaev, Al. L. Efros, I. A. Merkulov, V. Stavarache, D. Reuter, and A. Wieck, Phys. Rev. Lett. 96, 227401 (2006).

[7] T. A. Kennedy, A. Shabaev, M. Scheibner, Al. L. Efros, A. S. Bracker, and D. Gammon, Phys. Rev. B 73, 045307 (2006).

[8] Sophia E. Economou, L. J. Sham, Yanwen Wu, and D. G. Steel, Phys. Rev. B 74, 205415 (2006).

[9] T. Takagahara, J. Opt. Soc. Am. B 27, A46 (2010).

[10] Pochung Chen, C. Piermarocchi, L. J. Sham, D. Gammon, and D. G. Steel, Phys. Rev. B 69, 075320 (2004).

[11] D. Press, T. D. Ladd, B. Zhang, and Y. Yamamoto, Nature 456, 218 (2008).

[12] A. Greilich, Sophia E. Economou, S. Spatzek, D. R. Yakovlev, D. Reuter, A. D. Wieck, T. L. Reinecke, and M. Bayer, Nat. Phys. 5, 262 (2009).

[13] J. Javanainen, Europhys. Lett. 17, 407 (1992).

[14] Sophia E. Economou, Ren-Bao Liu, L. J. Sham, and D. G. Steel, Phys. Rev. B 71, 195327 (2005).

[15] S. Tomimoto, K. Kawana, A. Murakami, and Y. Masumoto, in preparation.

[16] Y. Masumoto, I. V. Ignatiev, K. Nishibayashi, T. Okuno, S. Yu. Verbin, and I. A. Yugova, J. Lumin. 108, 177 (2004).

[17] I. V. Ignatiev, T. Okuno, S. Yu. Verbin, I. A. Yugova, and Y. Masumoto, Physica E 17, 365 (2003).

[18] I. A. Yugova, I. Ya. Gerlovin, V. G. Davydov, I. V. Ignatiev, I. E. Kozin, H. W. Ren, M. Sugisaki, S. Sugou, and Y. Masumoto, Phys. Rev. B 66, 235312 (2002).

[19] M. Ikezawa, B. Pal, Y. Masumoto, I. V. Ignatiev, S. Yu. Verbin, and I. Ya. Gerlovin, Phys. Rev. B 72, 153302 (2005).

[20] I. Ya Gerlovin, I. V. Ignatiev, I. A. Yugova, and Y. Masumoto, Opt. Spectroscopy 104, 577 (2008). 